LETTER TO JMG

\title{
More evidence for non-maternal inheritance of mitochondrial DNA?
}

\author{
H-J Bandelt, Q-P Kong, W Parson, A Salas
}

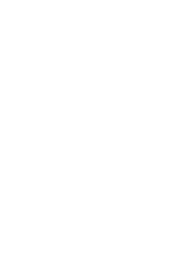

J Med Genet 2005;42:957-960. doi: 10.1136/jimg.2005.033589

Background: A single case of paternal co-transmission of mitochondrial DNA (mtDNA) in humans has been reported so far.

Objective: To find potential instances of non-maternal inheritance of mtDNA.

Methods: Published medical case studies (of single patients) were searched for irregular mtDNA patterns by comparing the given haplotype information for different clones or tissues with the worldwide mtDNA database as known to date-a method that has proved robust and reliable for the detection of flawed mtDNA sequence data.

Results: More than 20 studies were found reporting clear cut instances with mtDNAs of different ancestries in single individuals. As examples, cases are reviewed from recent published reports which, at face value, may be taken as evidence for paternal inheritance of mtDNA or recombination.

Conclusions: Multiple types (or recombinant types) of quite dissimilar mitochondrial DNA from different parts of the known mtDNA phylogeny are often reported in single individuals. From re-analyses and corrigenda of forensic mtDNA data, it is apparent that the phenomenon of mixed or mosaic mtDNA can be ascribed solely to contamination and sample mix up.

l: the past few years some exciting claims have been made about the mode of inheritance of mtDNA as well as the role of mtDNA in the pathogenesis of several human diseases. However, early attempts to show that mtDNA could undergo recombination in populations were based on misread ${ }^{12}$ or flawed data and unjustified premises about the mutational process, ${ }^{3}{ }^{4}$ or biased data collection, inadequate and misapplied statistics, and technical and logical errors. ${ }^{5-9}$ The observation that paternally inherited mtDNA was present in the muscle tissues of a Danish patient suffering from a mitochondrial myopathy ${ }^{10}$ is most remarkable but appears at present to be an isolated phenomenon, and has neither been confirmed in another laboratory nor found in other cases of sporadic myopathies. ${ }^{11-13}$ Most recently, ${ }^{14}$ it was reported that mtDNA recombination in singular muscle tissue had been demonstrated in vivo in the Danish myopathy patient. ${ }^{10}$ One way of searching for further potential instances of abnormal inheritance of mtDNA is to explore the rich medical genetics literature, where mtDNA information is generated on a case by case basis and recorded mutation by mutation-though sadly without taking the emerging mtDNA phylogeny into account. ${ }^{15}$

\section{METHODS}

In our examination we employed in principle all mtDNA sequences published so far $(>2000$ coding region sequences and $>30000$ partial control region sequences). The complete sequences can be hierarchically organised in a worldwide mtDNA phylogeny, the major branches (clades) of which are referred to as haplogroups, encoded in a hierarchical manner. ${ }^{16-20}$

\section{RESULTS}

Paternal m+DNA in Klinefelter's syndrome?

A case of abnormal mtDNA inheritance, which has apparently not attracted much attention in the scientific community, was reported in cases of Klinefelter's syndrome, ${ }^{21}$ where a possible interaction of the sex chromosome and mtDNA was hypothesised. In that study, mtDNA was analysed in eight Klinefelter males, seven from the USA and one from Japan, for the variation in the first hypervariable segment (HVS-I) and the second hypervariable segment (HVS-II) of the mtDNA control region. Most astonishingly, all seven American samples turned out to have identical HVS-I and HVS-II sequences outside the long C stretches (which is highly improbable for unrelated African American individuals) and even shared the same two heteroplasmies (table l, No 1). Puzzlingly, the Japanese Klinefelter individual (table 1, No 2) had almost the same array of mutations, but his mother (table 1, No 3) showed a number of different mutations, especially in HVS-II.

Note that the rCRS $^{22}$ nucleotide at position 223 was reported incorrectly as G (instead of T) and the "number of polymorphism" for the "normal Japanese" sample (of size 60) was inverted for most positions in HVS-II. ${ }^{21}$ The high level of polymorphism (34/60) for position 227 is most implausible in view of the corresponding value $(1 / 373)$ in two sets of Japanese data. ${ }^{23}{ }^{24}$ In any case, most of the nucleotide positions listed in table 1 were also claimed to be polymorphic (to various degrees) in the "normal Japanese". ${ }^{21}$ This is extraordinary in that, besides position 227, only position 263 has been found to be polymorphic in published Japanese data. Also worldwide, most of those positions are extremely conservative. In fact, the 10 positions $16060,16089,16208,16384,80,120,126,223,254$, and 299 were all found unvaried in the MITOMAP (http://www.mitomap.org/) and SWGDAM (http://www.fbi.gov/hq/lab/fsc/ backissu/april2002/millerl.htm) databases, except for one incorrectly recorded entry (16089) in MITOMAP, whereas 16042,227 , and 263 are known to be polymorphic to a very minor degree. In the Klinefelter data, ${ }^{21}$ the latter three positions solely show transitions but the former 10 positions all show transversions (table 1 ).

Some of these unexpected variants can be attributed to misinterpretation of the sequence electropherograms, which were generated using the ABI PRISM dye terminator cycle

Abbreviations: HVS-I, first hypervariable segment; HVS-II, second hypervariable segment; mtDNA, mitochondrial DNA; rCRS, revised Cambridge reference sequence 
Table 1 mtDNA sequences reported by Oikawa et al $(2002)^{21}$

Haplotype*

G16042A G16060K G16089K G16208T G16384A C80T A126C A263G

G16042A G16060K G16089K G16208T G16384A C80G A126C A263G

G16042A G16060T G16089T G16384C C80T C120G T223G A227G T254G A263G C299G

*Relative to the revised Cambridge reference sequence (rCRS; Andrews et al (1999) ${ }^{22}$ ): regions 16024-16400,

30-400; long $C$ stretches disregarded; $K=G / T$.

sequencing ready reaction kit and AmpliTag FS DNA polymerase. This version of sequencing chemistry is known to produce the frequently observed phenomenon that G peaks, 3' adjacent to A peaks, are displayed with significantly reduced peak height or may even vanish. ${ }^{25}$ This applies to positions 16060 and 16208, which are directly located 3' to adenosines (at 16059 and 16207). Depending on the background signal height these $G$ positions may then have been difficult to assign, although reverse sequencing, when applied, would correctly identify such positions.

The variation in the region 16184-16197 which includes the C stretch of HVS-I is also remarkable in these data. Although the first seven sequences are identical in HVS-I outside this stretch, all nine individuals differ within the stretch, yielding a peculiar pattern of mutations never

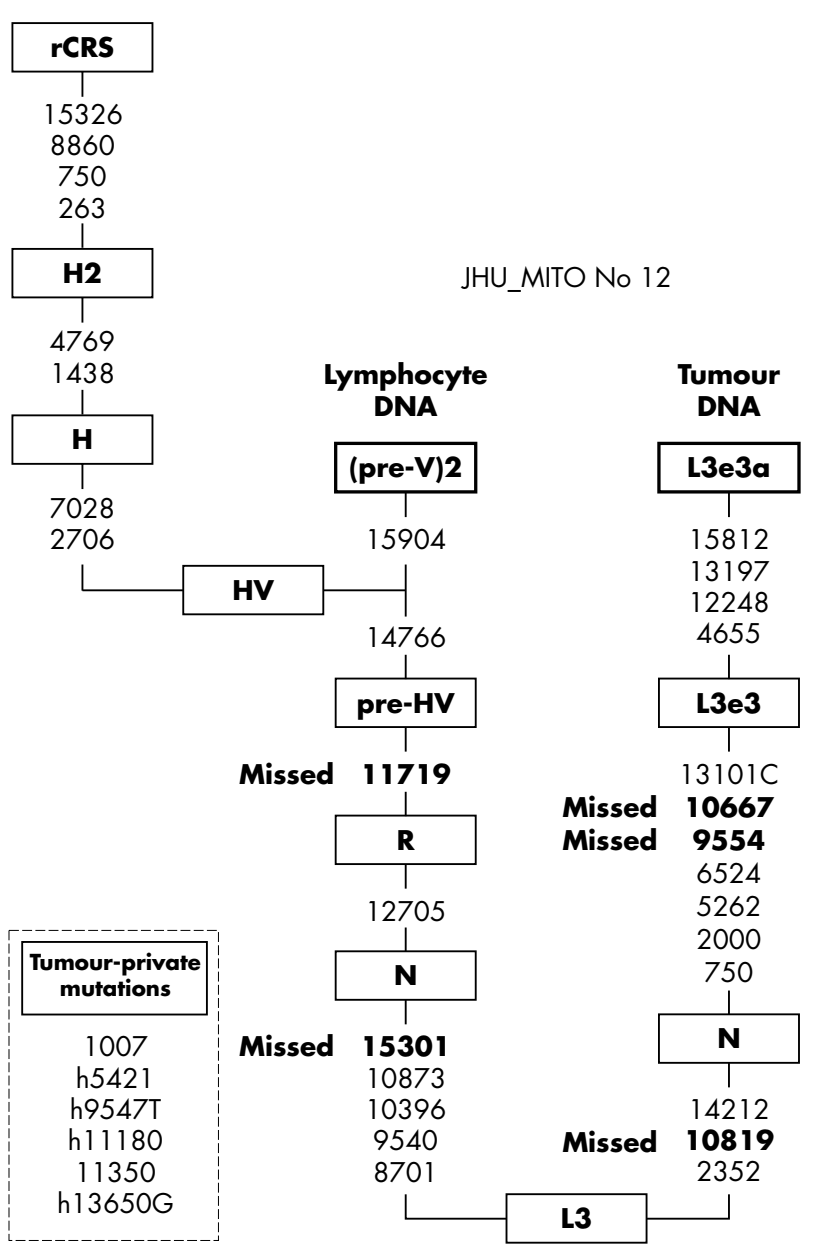

Figure 1 Coding region sites distinguishing two particular lineages from haplogroups (pre-V)2 and L3e 3 assigned to one individual (Maitra et al, 2004 ${ }^{28}$ ). Prefix $\mathrm{h}$ indicates heteroplasmy and a suffix designates a transversion. observed before. Similarly, the pattern in the region 303317 covering the C-stretch(es) of HVS-II with novel mutations is unusual. The chromatograms would rather indicate that length heteroplasmies were not properly recognised and erroneously interpreted as homoplasmic or heteroplasmic mutations in the $\mathrm{C}$ stretch. This is more likely to reflect additional sequencing problems associated with the C stretch, so that these differences would not necessarily prove that different mtDNA samples had actually been amplified.

We are thus seeing a very clear pattern of artificial mutations, as transversions are relatively rare in human mtDNA, especially in HVS-II, and thus signpost phantom mutations. ${ }^{26} 27$ In summary, the Klinefelter data ${ }^{21}$ bear multiple artefacts and were probably the combined result of biochemical problems (dye blobs and so on), poor quality and readability, and sample mix up or contamination. Whatever the specific causes of these patterns might be, these flawed data could not support statements such as "...we could conclude that strict maternal inheritance is disturbed in the generic of offspring with Klinefelter's syndrome" ${ }^{21}$

\section{Multiple somatic mutations in mtDNA?}

In cancer research, interest in mtDNA focuses on the question of whether somatic mutations accumulate in tumours. Recently, as many as 24 somatic mutations were observed in a single tumour of primary lung cancer (JHU_MITO No 12, supplementary table $3^{28}$ ), "providing additional evidence for a 'mitochondrial DNA mutator' phenotype". ${ }^{28}$ These mutations comprise four heteroplasmic and 20 homoplasmic mutations, 18 of which are, however, known to belong to an evolutionary pathway in the mtDNA phylogeny (fig 1). This mutational path separates haplogroup (pre-V)2 mtDNA $^{18}$ from a particular branch of haplogroup L3e3 mtDNA. ${ }^{29-31}$ Note that the MitoChip tool employed ${ }^{28}$ was unable to detect more than $80 \%$ of the mutations-though this detection rate was still sufficient to infer the pathway unambiguously. Clearly we are seeing here not somatic mutations (except possibly those four heteroplasmic mutations) but two mtDNA lineages stemming from different individuals that were, however, attributed to the same patient.

Another recent paper $^{32}$ aimed to demonstrate marked mtDNA heterogeneity among individual $\mathrm{CD}_{3} 4^{+}$clones from adult bone marrow. In particular, the predominant "aggregate" HVS-I and HVS-II sequence of many clones from the bone marrow donor No 2 actually matches (within 1602416365 and 73-340) a sequence (listed as USA.CAU.000339 in the SWGDAM database) that evidently belongs to the North African haplogroup U6al. ${ }^{17}$ One of the $\mathrm{CD} 34^{+}$clones from the same donor, however, gave a totally different sequence with another specific mutational motif (for example, CB155 and FO156 from a Japanese dataset ${ }^{24}$ or KOR.ASN.000124 and JPN.ASN.000041 from the SWGDAM database), which qualifies this sequence as a member of the East Asian haplogroup D4c. ${ }^{33}$ In another case, ${ }^{34}$ the mtDNA sequence of a single $\mathrm{CD}_{3} 4^{+}$clone from cord blood donor No 1 showed a pattern extremely distinct from the aggregate cord blood 
mtDNA and other $\mathrm{CD} 34^{+}$clones. Thus the aggregate sequence and the deviant sequence harbour the motifs of two distinct Indian subhaplogroups of haplogroup M (A73G T146C T195A A263G T489C 522-523del 16166del C16223T T16519C versus A73G A153G A263G C463T T485C T489C C16223T; authors' unpublished data). Although it was contended that "to prevent DNA cross-contamination, special precautions were taken", 32 it seems that these "precautions" were not sufficient. Two step nested polymerase chain reaction (PCR), as applied in these cases, bears an increased risk of allowing contaminant mtDNA to enter the process.

A study of mtDNA control region mutations in patients with oesophageal squamous cell carcinoma ${ }^{35}$ offers an interesting case of apparent recombinant mtDNA. The mtDNA found in the tumour sample of case 20 (C16185T C16223T C16260T T16298C) clearly indicates that this is an East Asian haplogroup Z sequence, ${ }^{16}$ whereas the blood sample (C16256T C16270T Al6399G) points to the European haplogroup U5al. ${ }^{19} 20$ In contrast, no single discriminating mutation was recorded for HVS-II, despite the fact that the HVS-II mutation motif T152C 249d T489C would clearly separate those two haplogroups. Thus we infer that either the blood mtDNA or the tumour mtDNA must constitute a recombinant type. The reported data ${ }^{35}$ contain yet another case of totally different mtDNA lineages in one patient, and there are plenty of further cases from cancer research where mixed or recombinant mtDNA samples in single patients can be inferred in the same way as outlined above.

In summary, previous screening of mtDNA data from evolutionary and forensic studies has provided a rich record of obviously flawed or doubtful results that would directly affect the question of whether mtDNA may occasionally be inherited non-maternally. Clear cut artificial recombinants between separately amplified segments can easily be detected through focused database searches and phylogenetic analysis. ${ }^{36}$ For instance, the SWGDAM database-a forensic mtDNA database that went online in 2002-contained at least six obvious instances of artificial recombination, ${ }^{3637}$ only four of which have been corrected so far through two partial revisions of the database for transcription errors. ${ }^{38} 39$ This could indicate that some recombinants had been generated in the laboratory through sample mix up or contamination and are therefore unrecognisable by mere re-reading of sequencer outputs. The German database " $\mathrm{D}$ Loop-BASE" (http://www.d-loop-base.de/) suffered from massive artificial recombination and all kinds of other problems, ${ }^{40}$ so that it eventually had to go offline in early 2004.

Have we really discovered new cases of non-maternal inheritance of mtDNA? The Klinefelter $\operatorname{case}^{21}$ can be dismissed as the result of an obvious sequencing disaster. The other instances ${ }^{28} 3234$ discussed above indeed point to different sources of the mtDNAs that were attributed to single patients. In one cancer patient, ${ }^{35}$ one compound haplotype is even composed from HVS-I and HVS-II stemming from different mtDNAs. To explain these findings, however, one does not have to invoke novel mechanisms of mtDNA inheritance. The lesson learnt from forensic mtDNA databases offers a much more straightforward explanationnamely, the mechanism of laboratory artefacts.

The recent study ${ }^{14}$ about seeming recombination of mtDNA in the Danish patient should give researchers a hint that recombination needs to be considered as a potential factor in their studies-but then artificial recombination should come to mind first. Among other questionable aspects of that study, ${ }^{14}$ we observe that the methodological approach employed (single molecule PCR) is in fact extremely prone to contamination (owing to the minimal amounts of DNA that were used); moreover, the highly statistically significant
mtDNA recombination hotspots $\mathrm{B}$ and $\mathrm{C}$ bound familiar mtDNA segments generated with standard primer pairs (as applied in human population genetics). Therefore, there is room here for much scepticism.

The cohorts of published artefacts in forensic databases $^{27} 36374041$ suggest that the most natural reason for seeing totally different mtDNAs or mosaic compound haplotypes in a single individual is casual handling and mis-sequencing of samples in the laboratory. It seems that the chances for artificial recombination and the possibility of other systematic errors in mtDNA analyses are notoriously underestimated. With solid complete sequencing studies, there is increasing evidence that there is absolutely no sign of natural recombination. ${ }^{729}$ For example, the HVS-I and HVSII status was a good predictor of the coding region variation in cases of frequent HVS-I and HVS-II haplotypes, notwithstanding occasional single recurrent mutations in HVS-I and HVS-II. ${ }^{42}$ On the other hand, poor laboratory work executed on large parts of the coding region yielded very clear signals of artefacts..$^{43}$ In summary, the point is that the mtDNA phylogeny mirrors the population history during thousands of years, a period that should be long enough to generate a signal of recombination, if any is present. Any population harbours phylogenetically distant mtDNA haplotypes, so that there is in general about a $50 \%$ chance of having such distant mtDNAs involved in a recombination event-whether real or not. It is then evident that the frivolous statement "recombination is difficult to detect in population genetic data, even if it is occurring at appreciable frequencies" ${ }^{\prime 45}$ is plainly wrong.

It has been said that "special claims require special evidence". ${ }^{46}$ Non-maternal inheritance of mtDNA would certainly be very special. This means that for every result that called the dogma of maternal inheritance of mtDNA into question one would need independent extraction, amplification, and sequencing in another laboratory-in analogy to the situation with ancient DNA, where independent replication is required. ${ }^{47}$ This clearly holds for all cases of (seeming) paternal inheritance or blocks of multiple somatic mutations in patients. We agree that "systematic haplotype analyses of large cohorts of patients with sporadic mtDNA mutations and healthy individuals are therefore warranted to unravel this enigma". ${ }^{48}$ However, it appears to be even more important to unravel the causes of laboratory artefacts that might lead to premature claims of mitochondrial association with certain diseases $^{49}$ or of sporadic non-maternal inheritance of mtDNA. Any attempt to propose recombination of mtDNA in such cases should also explain why the worldwide mtDNA phylogeny faithfully mirrors the non-recombinant nature of this genome.

\section{Authors' affiliations \\ H-J Bandelt, Fachbereich Mathematik, Universität Hamburg, Hamburg, Germany \\ Q-P Kong, Key Laboratory of Cellular and Molecular Evolution and Molecular Biology of Domestic Animals, Kunming Institute of Zoology, Chinese Academy of Sciences, Kunming, Yunnan, China \\ W Parson, Institute of Legal Medicine, Innsbruck Medical University, Innsbruck, Austria \\ A Salas, Unidad de Genética, Instituto de Medicina Legal, Facultad de Medicina, Santiago de Compostela, Galicia, Spain \\ Competing interests: none declared}

Correspondence to: Dr Hans-Jürgen Bandelt, Fachbereich Mathematik, Universität Hamburg, Bundesstr 55, 20146 Hamburg, Germany; bandel+@math.uni-hamburg.de

Received 1 April 2005

Revised version received 15 May 2005

Accepted for publication 17 May 2005

Published Online First 27 May 2005 


\section{REFERENCES}

1 Hagelberg E, Goldman N, Lio P, Whelan S, Schiefenhovel W, Clegg JB, Bowden DK. Evidence for mitochondrial DNA recombination in a human population of island Melanesia. Proc R Soc London Ser B Biol Sci 1999:266:485-92.

2 Hagelberg E, Goldman N, Lio P, Whelan S, Schiefenhovel W, Clegg JB, Bowden DK. Evidence for mitochondrial DNA recombination in a human population of island Melanesia: correction. Proc R Soc London Ser B Biol Sci 2000;267:1595-6.

3 Eyre-Walker A, Smith NH, Smith JM. How clonal are human mitochondria? Proc R Soc London Ser B Biol Sci 1999;266:477-83

4 Macaulay V, Richards M, Sykes B. Mitochondrial DNA recombination - no need to panic. Proc R Soc London Ser B Biol Sci 1999;266:2037-9.

5 Awadalla P, Eyre-Walker A, Smith JM. Linkage disequilibrium and recombination in hominid mitochondrial DNA. Science 1999;286:2524-5.

6 Kivisild T, Villems R. Questioning evidence for recombination in human mitochondrial DNA. Science 2000;288:1931a

7 Elson JL, Andrews RM, Chinnery PF, Lightowlers RN, Turnbull DM, Howell N. Analysis of European mtDNAs for recombination. Am J Hum Genet 2001;68:145-53.

8 Piganeau G, Eyre-Walker A. A reanalysis of the indirect evidence for recombination in human mitochondrial DNA. Heredity 2004;92:282-8.

9 Hedrick P, Kumar S. Mutation and linkage disequilibrium in human mtDNA. Eur J Hum Genet 2001;9:969-72.

10 Schwartz M, Vissing J. Paternal inheritance of mitochondrial DNA. N Engl J Med 2002;347:576-80

11 Filosto M, Mancuso M, Vives-Bauza C, Vila MR, Shanske S, Hirano M, Andreu AL, DiMauro S. Lack of paternal inheritance of muscle mitochondrial DNA in sporadic mitochondrial myopathies. Ann Neurol 2003:54:524-6.

12 Taylor RW, McDonnell MT, Blakely EL, Chinnery PF, Taylor GA, Howell N, Zeviani M, Briem E, Carrara F, Turnbull DM. Genotypes from patients indicate no paternal mitochondrial DNA contribution. Ann Neurol 2003;54:521-4.

13 Schwartz M, Vissing J. No evidence for paternal inheritance of mtDNA in patients with sporadic mtDNA mutations. J Neurol Sci 2004;218:99-101.

14 Kraytsberg Y, Schwartz M, Brown TA, Ebralidse K, Kunz WS, Clayton DA, Vissing J, Khrapko K. Recombination of human mitochondrial DNA. Science 2004;304:981.

15 Herrnstadt C, Howell N. An evolutionary perspective on pathogenic mtDNA mutations: haplogroup associations of clinical disorders. Mitochondrion 2004;4:791-8

16 Kong Q-P, Yao Y-G, Sun C, Bandelt H-J, Zhu C-L, Zhang Y-P. Phylogeny of East Asian mitochondrial DNA lineages inferred from complete sequences. Am J Hum Genet 2003:73:671-6; erratum, 75:157.

17 Maca-Meyer N, Gonzalez AM, Pestano J, Flores C, Larruga JM, Cabrera VC Mitochondrial DNA transit between West Asia and North Africa inferred from U6 phylogeography. BMC Genetics 2003;4:15.

18 Achilli A, Rengo C, Magri C, Battaglia V, Olivieri A, Scozzari R, Cruciani F, Zeviani M, Briem E, Carelli V, Moral P, Dugoujon JM, Roostalu U, Loogvali EL, Kivisild T, Bandelt HJ, Richards M, Villems R, Santachiara-Benerecetti AS, Semino O, Torroni A. The molecular dissection of m+DNA haplogroup $H$ confirms that the Franco-Cantabrian glacial refuge was a major source for the European gene pool. Am J Hum Genet 2004:75:910-18.

19 Achilli A, Rengo C, Battaglia V, Pala M, Olivieri A, Fornarino S, Magri C, Scozzari R, Babudri N, Santachiara-Benerecetti AS, Bandelt HJ, Semino O, Torroni A. Saami and Berbers - an unexpected mitochondrial DNA link. Am J Hum Genet 2005;76:883-6.

20 Palanichamy M, Sun C, Agrawal S, Bandelt HJ, Kong QP, Khan F, Wang CY, Chaudhuri TK, Palla V, Zhang YP. Phylogeny of mtDNA macrohaplogroup $N$ in India based on complete sequencing: implications for the peopling of South Asia. Am J Hum Genet 2004;75:966-78.

21 Oikawa H, Tun Z, Young DR, Ozawa H, Yamazaki K, Tanaka E, Honda K. The specific mitochondrial DNA polymorphism found in Klinefelter's syndrome. Biochem Biophys Res Commun 2002;297:341-5

22 Andrews RM, Kubacka I, Chinnery PF, Lightowlers RN, Turnbull DM, Howell N. Reanalysis and revision of the Cambridge reference sequence for human mitochondrial DNA. Nat Genet 1999:23:147.

23 Monson KL, Miller KW, Wilson MR, DiZinno JA, Budowle B. The mtDNA population database: an integrated software and database resource for forensic comparison. Forensic Sci Commun 2002;4:2

24 Maruyama S, Minaguchi K, Saitou N. Sequence polymorphisms of the mitochondrial DNA control region and phylogenetic analysis of mtDNA lineages in the Japanese population. Int J Legal Med 2003;117:218-25.

25 Parson W, Parsons TJ, Scheithaver R, Holland MM. Population data for 101 Austrian Caucasian mitochondrial DNA d-loop sequences: application of
mtDNA sequence analysis to a forensic case. Int J Legal Med 1998;111:124-32.

26 Bandelt H-J, Quintana-Murci L, Salas A, Macaulay V. The fingerprint of phantom mutations in mitochondrial DNA data. Am J Hum Genet 2002:71:1150-60.

27 Yao Y-G, Bravi CM, Bandelt $\mathrm{H}-J$. A call for mtDNA data quality control in forensic science. Forensic Sci Int 2004;141:1-6.

28 Maitra A, Cohen Y, Gillespie SE, Mambo E, Fukushima N, Hoque MO, Shah N, Goggins M, Califano J, Sidransky D, Chakravarti A. The human MitoChip: a high-throughput sequencing microarray for mitochondrial mutation detection. Genome Res 2004; 14:812-19.

29 Ingman M, Kaessmann H, Paabo S, Gyllensten U. Mitochondrial genome variation and the origin of modern humans. Nature 2000:408:708-13.

30 Bandelt H-J, Alves-Silva J, Guimaraes P, Santos MS, Brehm A, Pereira L, Coppa A, Larruga JM, Rengo C, Scozzari R, Torroni A, Prata MJ, Amorim A, Prado VF, Pena SD. Phylogeography of the human mitochondrial haplogroup L3e: a snapshot of African prehistory and Atlantic slave trade. Ann Hum Genet 2001;65:549-63.

31 Herrnstadt C, Elson JL, Fahy E, Preston G, Turnbull DM, Anderson C, Ghosh SS, Olefsky JM, Beal MF, Davis RE, Howell N. Reduced-mediannetwork analysis of complete mitochondrial DNA coding-region sequences for the major African, Asian, and European haplogroups. Am J Hum Genet 2003;70:1152-71. Correction, 71:448-9.

32 Shin MG, Kajigaya S, Tarnowka M, McCoy JP, Levin BC, Young NS. Mitochondrial DNA sequence heterogeneity in circulating normal human CD34 cells and granulocytes. Blood 2004b;103:4466-77.

33 Comas D, Plaza S, Wells RS, Yuldaseva N, Lao O, Calafell F, Bertranpetit J. Admixture, migrations, and dispersals in Central Asia: evidence from maternal DNA lineages. Eur J Hum Genet 2004;12:495-504.

34 Shin MG, Kajigaya S, McCoy JP, Levin BC, Young NS. Marked mitochondrial DNA sequence heterogeneity in single CD34+ cell clones from normal adult bone marrow. Blood 2004a; 103:553-61.

35 Abnet CC, Huppi K, Carrera A, Armistead D, McKenney K, Hu N, Tang ZZ, Taylor PR, Dawsey SM. Control region mutations and the "common deletion" are frequent in the mitochondrial DNA of patients with esophageal squamous cell carcinoma. BMC Cancer 2004;4:30.

36 Bandelt H-J, Salas A, Lutz-Bonengel S. Artificial recombination in forensic mtDNA population databases. Int J Legal Med 2004b;1 18:267-73.

37 Bandelt $\mathrm{H}-\mathrm{J}$, Salas A, Bravi C. Problems in FBI mtDNA database. Science 2004;305: 1401-4.

38 Budowle B, Polanskey D, Allard MW, Chakraborty R. Addressing the use of phylogenetics for identification of sequences in error in the SWGDAM mitochondrial DNA database. J Forensic Sci 2004;49:1-6.

39 Polanskey D, Budowle B. Summary of the findings of a quality review of the Scientific Working Group on DNA analysis methods mitochondrial DNA database. Forensic Sci Commun 2005;7:1.

40 Bandelt H-J, Parson W. Fehlerquellen mitochondrialer DNA-Datensatze und Evalution der mtDNA-Datenbank "D-Loop-BASE". Rechtsmedizin 2004; 14:251-7.

41 Poetsch M, Wittig H, Krause D, Lignitz E. Corrigendum to "Mitochondrial diversity of a northeast German population sample". Forensic Sci Int 2004; 145:73-7.

42 Coble MD, Just RS, $\mathrm{O}^{\prime}$ Callaghan JE, Letmanyi IH, Peterson CT, Irwin JA, Parsons TJ. Single nucleotide polymorphisms over the entire mtDNA genome that increase the power of forensic testing in Caucasians. Int $J$ Legal Med 2004; 118:137-46

43 Yao Y-G, Macaulay V, Kivisild T, Zhang Y-P, Bandelt H-J. To trust or not to trust an idiosyncratic mitochondrial data set. Am J Hum Genet 2003:72:1341-6.

44 Bandelt H-J, Achilli A, Kong Q-P, Salas A, Lutz-Bonengel S, Sun C, Zhang Y-P, Torroni A, Yao Y-G. Low "penetrance" of phylogenetic knowledge in mitochondrial disease studies. Biochem Biophys Res Commun 2005:331:1301-9.

45 Ladoukakis ED, Eyre-Walker A. Direct evidence of recombination in human mitochondrial DNA. Heredity 2004;93:321.

46 Wallis GP. Do animal mitochondrial genomes recombine? Trends Ecol Evol 1999;14:209-10.

47 Cooper AR, Poinar H. Ancient DNA: do it right or not at all. Science 2000;289:1139.

48 Schwartz M, Vissing J. New patterns of inheritance in mitochondrial disease: Biochem Biophys Res Commun 2003;310:247-51.

49 Bandelt H-J, Yao Y-G, Kivisild T. Mitochondrial genes and schizophrenia. Schizophr Res 2005;72:267-9. 